\title{
Certificación en los servicios ciudadanos: una experiencia práctica
}

Citizen services certification: a practical experience

\author{
Julián ARROYo ÁLVAREZ \\ Ayuntamiento de Valladolid (España) \\ julian@infor.uva.es
}

\begin{abstract}
Resumen
Durante el año 2011, el Ayuntamiento de Valladolid ha llevado a cabo la puesta en marcha de un proceso de modernización administrativa, basado en la certificación electrónica en la prestación de servicios a los ciudadanos y que tiene su reflejo externo en tres pilares fundamentales: la sede electrónica, el portal web y el portal de participación. Este artículo describe el resultado de este trabajo y cuáles son los objetivos que han guiado su desarrollo.
\end{abstract}

Palabras clave: Administración electrónica. Gobierno local. Modernización administrativa. Valladolid. (Ayuntamiento).

\section{Introducción}

El Plan Estratégico de la ciudad, Valladolid hacia el 2016, ya presentaba entre sus objetivos la promoción del gobierno electrónico en el municipio. Además de este plan interno, la aprobación de la Ley de Acceso Electrónico de los Ciudadanos a los Servicios Públicos (LAECSP) ha servido de impulso para abordar un proyecto de implantación de la administración electrónica, que como proceso dinámico se dirige al concepto de "Ayuntamiento de Valladolid: ayuntamiento abierto".

Este proyecto de implantación se ha configurado sobre los siguientes puntos: empleo de informática y comunicaciones, cambios organizativos, nuevas aptitudes, mejora de los servicios, mejora de los procesos democráticos, y apoyo a la formación de las políticas públicas

Es éste, por tanto, un claro ejemplo de un proyecto de gestión del cambio, que va más allá de la mera implantación de tecnologías en una administración pública, que ha requerido, y aún requiere, un proceso de colaboración públicoprivada, en la que la parte pública debe aportar una fuerte implicación de toda la organización, dirigida con un fuerte liderazgo; mientras, la parte privada debe aportar también una fuerte implicación, con gran capacidad de adaptación, así como unos profundos conocimientos de gestión de procesos y, por supuesto, tecnológi-

\begin{abstract}
In 2011, Valladolid City Council carried out a modernization process in its administrative tasks. It was focused on providing citizen services using electronic certificates and it was based in three main points: virtual office, web portal and participation portal. This article describes the results obtained from this work and what were the objectives that have leaded this development.
\end{abstract}

Keywords: e-Government. Local government. Government modernization. Valladolid (city council).

cos. En definitiva, estamos hablando de un proyecto que supone un importante esfuerzo colectivo.

\section{Planteamiento}

Junto a la formación de estos equipos de trabajo -y de forma simultánea- se elaboró un plan de actuación que buscaba ser muy sencillo (cuanto más simple, más fácil de implantar), muy visible y muy acotado, tanto en las materias a intervenir como en el tiempo a ejecutarse.

Por tanto, el plan incluía como objetivos cuatro puntos básicos. El primero de ellos era el cumplimiento de la legislación en vigor, que obligaba al Ayuntamiento a poner en marcha una experiencia de administración electrónica sólida. No nos referimos únicamente a la Ley de Acceso Electrónico de los Ciudadanos a los Servicios Públicos (Ley 11/2007), sino también a lo establecido en la Ley de Contratos del Sector Público (Ley 30/2007) y su posterior texto refundido (RDL 3/2011), en lo que afecta a la existencia de un perfil del contratante de cada administración pública con una serie de obligaciones técnicas y legales, o a la Ley de Servicios de la Sociedad de la Información y Comercio Electrónico (Ley 34/2002) en lo relativo a la accesibilidad de los servicios web administrativos y la Ley de Reutilización de Información del Sector Público (Ley 37/2007), en la medida que la infor- 
mación generada por las administraciones debe ser facilitada por el mejor medio posible a los ciudadanos para su posterior explotación.

El segundo objetivo sería plasmar el concepto de Ayuntamiento abierto, en el sentido de dar mayor participación al ciudadano en la elaboración de políticas públicas y en el aumento de la transparencia en la gestión municipal. Este punto nace de la necesidad de acercar al ciudadano al gobierno de su territorio, como parte fundamental del mismo y propio de sociedades democráticas avanzadas.

Por una parte, se trata de aumentar la transparencia en el ejercicio del poder por parte de la administración; mientras que, por otra, esta misma administración pretende incorporar la participación del ciudadano en la formación de políticas públicas, de manera que el ejercicio de ese poder no se perciba como algo lejano, menos aún en una administración territorial tan cercana al ciudadano como es un ayuntamiento.

Además, la iniciativa Open Data, ya regulada por la legislación estatal, busca facilitar la compartición de la información manejada por las administraciones con sus ciudadanos (y ya pagada por ellos), sin más restricciones que las derivadas de la protección de los derechos personales.

Como tercer objetivo, nos encontramos con el ya mencionado cumplimiento del mandato establecido por el plan estratégico de la ciudad "Valladolid hacia el 2016", en lo que se refiere al fomento del gobierno electrónico como forma innovadora de toma de decisiones.

Y como último objetivo, aunque no menos destacable, estaría el hecho de que la página web municipal se había quedado obsoleta, tanto en servicios, como en tecnología y diseño gráfico. A pesar de ser una página tenida como referente en el sector de las administraciones locales desde su lanzamiento en 2002, el tiempo y la falta de un proyecto de renovación, hicieron que la presencia del Ayuntamiento de Valladolid en Internet fuera perdiendo atractivo con el paso del tiempo.

Uno de los principales defectos que tenía la anterior web municipal, y que se ha reenfocado desde la visión de la nueva plataforma electrónica, era la obligación de realizar un mantenimiento centralizado de la información, lo cual restaba inmediatez en la actualización de los datos y requería establecer un modelo excesivamente burocratizado en la gestión de los cambios a publicar.

Por otro lado, si bien ya existía un perfil del contratante de acuerdo a la normativa sobre contra- tos del sector público, sus publicaciones carecían de garantía legal y no hubieran podido superar una auditoría sobre este punto.

\section{Desarrollo del proyecto}

Podemos considerar que a la hora de afrontar el proyecto de administración electrónica del Ayuntamiento de Valladolid, se establecieron una serie de puntos básicos que resultaron claves en el desarrollo.

\subsection{Certificación}

Primeramente, la idea era conseguir resultados, pudiendo reutilizar las mejores ideas que ya existiesen en el campo de la administración electrónica, consiguiendo ahorro de costes en el desarrollo de sistemas y aprovechando la experiencia previa de otras entidades. Así, se estableció que antes que desarrollar un nuevo sistema de certificación, partiendo de cero, se emplearía la plataforma @firma, puesta a disposición de todos los organismos públicos por la Administración General del Estado.

Esta plataforma que lidera el Ministerio de Política Territorial y Administraciones Públicas, proporciona herramientas que permiten la validación de múltiples certificados electrónicos, la práctica totalidad de los emitidos en España. Con estos certificados, entre ellos los contenidos en el Documento Nacional de Identidad Electrónico (DNle), se pueden firmar documentos dirigidos al Ayuntamiento de Valladolid con plena validez jurídica y sin necesidad de pasar por un registro municipal presencial.

También, la plataforma @firma dispone de una autoridad de sellado de tiempo, TS@, que garantiza la validez de los asientos en los registros administrativos de cualquier administración adherida; y, como otra pieza del engranaje para fomentar la cooperación e interrelación entre las administraciones públicas, se encuentra el Sistema de Aplicaciones y Redes para las Administraciones, más conocido como red SARA. Esta red privada segura entre administraciones, permite el intercambio de información y acceso a bases de datos de otras administraciones sin necesidad de infraestructura adicional.

\subsection{Portales web}

El segundo aspecto definido como básico, fue que la solución de cara al ciudadano debería consistir en un entorno web multiportal, de tal manera que la información se almacenase en un repositorio de datos común y pudiese ser empleada en una u otra parte sin el más mínimo 
problema de interoperabilidad, facilitando la experiencia del usuariotres

De este modo, se definieron 3 portales diferentes, en función del uso requerido por los ciudadanos. Por un lado, estaría el portal web municipal (www.valladolid.es), dedicado a informar y dar servicios propios del Ayuntamiento sin funciones administrativas, y un nuevo concepto de portal web, denominado portal de participación, dedicado a fomentar la interacción con el ciudadano. Por otro lado, nos encontramos la sede electrónica, con dominio de Internet diferenciado (www.valladolid.gob.es), que satisface las exigencias de la LAESCP y se dedica a la publicación de información oficial municipal, así como habilita la iniciación de expedientes administrativos de forma totalmente telemática.

\subsubsection{Sede electrónica}

Más en detalle, la sede electrónica del Ayuntamiento de Valladolid (imagen mostrada en la figura 1) dispone de un tablón oficial de anuncios que sustituye al que existía de forma física anteriormente. En este tablón se publican tanto documentos internos, tales como edictos, ayudas y convocatorias de diferentes órganos municipales, como documentos de origen externo, procedentes de otras administraciones públicas u órganos judiciales. En cualquiera de los casos, todas las publicaciones cuentan con plena validez jurídica, al estar firmadas con el propio certificado electrónico de la sede y selladas a través del certificado de la autoridad de sellado de tiempo.

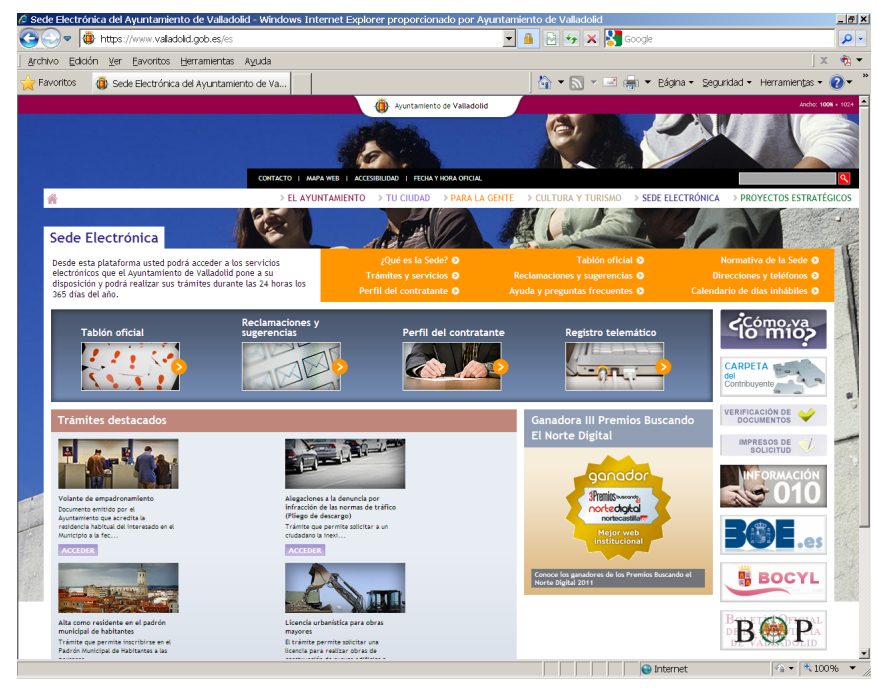

Figura 1. Imagen de la sede electrónica del Ayuntamiento de Valladolid

Esta sede electrónica tiene además otro servicio disponible, como es el' de registro electrónico, cuya puesta en marcha ha supuesto diversas actuaciones que van desde el establecimiento de un responsable administrativo, hasta el establecimiento de la plataforma de certificación para la identificación de los ciudadanos, pasando por una catalogación de todos los procedimientos que se pueden seguir en el Ayuntamiento, de tal manera que en la actualidad, se puede presentar de forma telemática casi cualquier instancia o solicitud en el ámbito municipal, junto con su documentación asociada.

Por otra parte, la carpeta ciudadana, implantada progresivamente con todos los trámites disponibles en la administración municipal, permite hacer un seguimiento de cualquier expediente abierto en el Ayuntamiento por parte de la persona que lo inició. Para ello, lógicamente, deberá identificarse por medio de un certificado electrónico de los admitidos por la sede electrónica.

Además de estos tres servicios, la sede electrónica sirve de soporte para la publicación del perfil del contratante, en donde aparecen todos los expedientes de contratación de la entidad en un formato que impide su manipulación sin dejar huellas informáticas y sirve también para implantar de forma sencilla el control del ciclo de vida de las contrataciones, acreditándose los cambios de estado mediante la combinación de una firma electrónica de sede y un sello de tiempo garantizado por la autoridad perteneciente al Gobierno Central.

\subsubsection{Portal web}

En cuanto al portal web municipal (imagen mostrada en la figura 2), éste se dedica a la publicación de todo tipo de información del municipio que sea de interés para el ciudadano. La información que aparece en este portal está clasificada siguiendo tres criterios diferentes de manera que aparezca en todas las páginas donde ésta tenga relación; se clasifica en función del órgano (concejalía o equivalente) para aquellos ciudadanos que quieran buscar la información a través de la estructura municipal interna ("el ayuntamiento"), en función de los temas tratados sin importar su competencia orgánica ("tu ciudad") y también en función de los colectivos destinatarios de la información o servicios ("para la gente"). Así, por ejemplo, una información sobre escuelas infantiles, estará vinculada al área de Bienestar Social y Familia y al área de Participación Ciudadana, que es donde se encuentra encuadrada orgánicamente, pero también a los temas "educación" y "servicios sociales" y al colectivo "familias".

Este portal cuenta también con una agenda de eventos de todo tipo a desarrollar en la ciudad, 
alimentada automáticamente desde fuentes de datos especializadas y una serie de servicios adicionales como un callejero con acceso a la cartografía oficial municipal, la consulta de los fondos documentales del Archivo Municipal, las incidencias de tráfico o un exitoso servicio de adopción de animales gestionado por el centro canino.

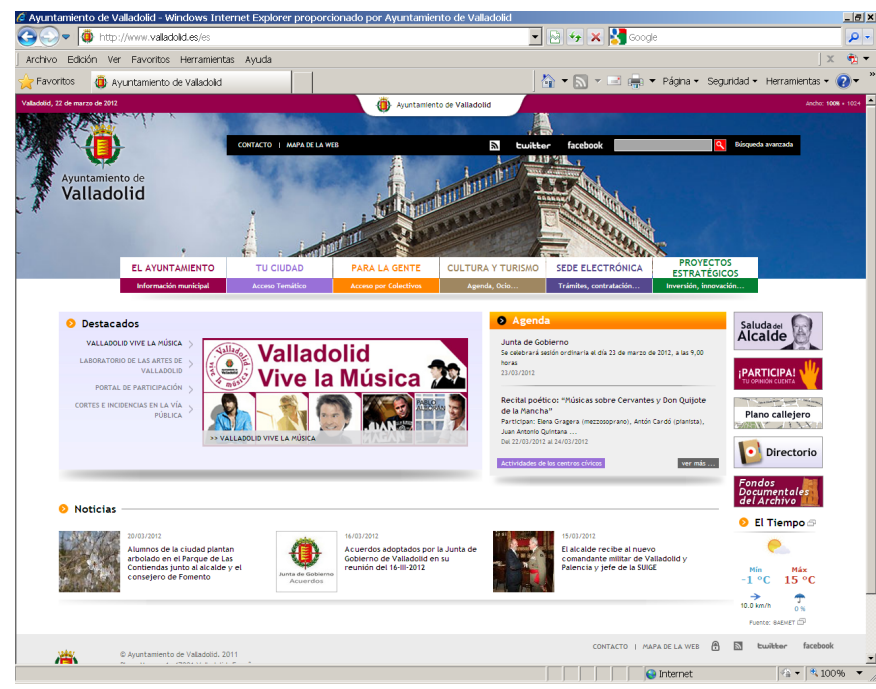

Figura 2. Imagen del nuevo portal web del Ayuntamiento de Valladolid

\subsubsection{Portal de participacion}

Por lo que se refiere al portal de participación (figura 3), se trata de un elemento fundamental en la estrategia de apertura del consistorio a los ciudadanos promovida por el Ayuntamiento de Valladolid. En una clara orientación hacia la web 2.0, los ciudadanos pueden interactuar como usuarios registrados y dar su opinión a través de tres herramientas disponibles.

La primera de estas herramientas son varios blogs mantenidos desde el propio ayuntamiento sobre diferentes aspectos de la vida municipal. Uno de ellos es el blog del alcalde, desde el cual el propio alcalde de la ciudad expone un tema de actualidad y lo somete a los comentarios de los ciudadanos. Otro de ellos es un blog de eventos culturales, "En tu corazón, Valladolid", como mecanismo de difusión de las diferentes actividades que se celebran habitualmente en la ciudad. También existe otro blog dedicado a la historia de la ciudad, visto a través de los documentos disponibles en el Archivo Municipal de Valladolid.

Es de destacar que la versatilidad del gestor documental que soporta la publicación web del portal, permite la creación y configuración de nuevos blogs en función de nuevas necesidades o demandas de algún colectivo en concreto.

Junto a estos blogs publicados desde el propio Ayuntamiento de Valladolid, también se incorpora un nuevo concepto, denominado planeta de blogs, que permite la publicación de otros blogs externos dentro del propio portal web municipal.

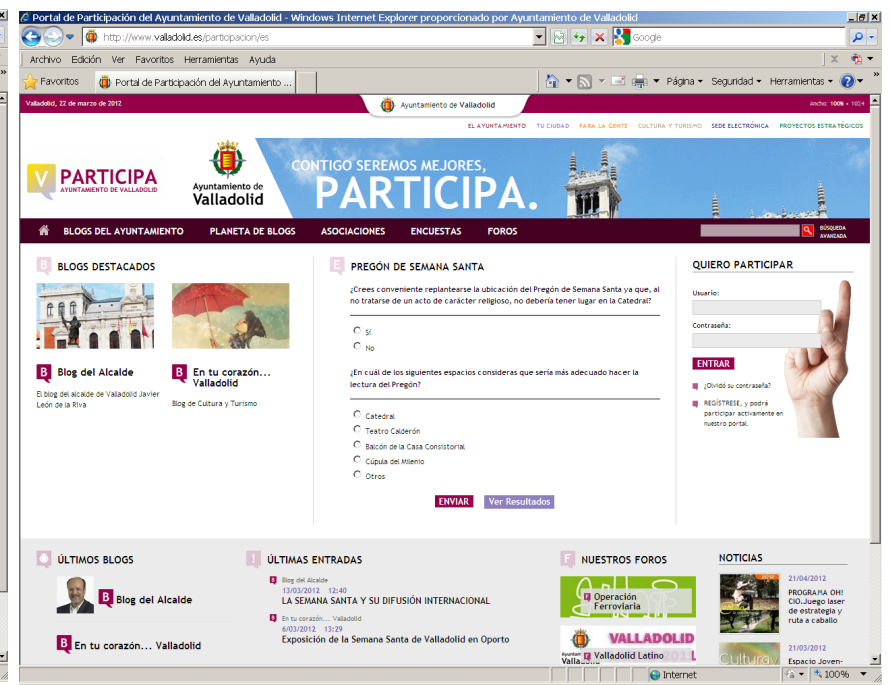

Figura 3. Imagen del portal de participación del Ayuntamiento de Valladolid

Este planeta de blogs acoge las publicaciones de blogueros que tengan alguna relación con Valladolid, den su permiso, y cuyas entradas se consideren de interés para los ciudadanos. Así, hay un blog dedicado a la semana santa de Valladolid, otro sobre las experiencias de un periodista vallisoletano en Noruega, otro sobre las ofertas y gangas que se pueden encontrar en los comercios de la ciudad y, recientemente, otro más sobre anécdotas relacionadas con la cultura e historia del municipio.

La segunda de las herramientas son unos foros de discusión sobre temas de actualidad de la ciudad. Aunque formalmente estos foros están ya en funcionamiento, está previsto que alcancen una mayor relación con el ciudadano a medida que vayan estando a disposición pública los datos relativos a la elaboración del nuevo plan general de ordenación urbana, actualmente en desarrollo.

Por último, la tercera de las herramientas es la posibilidad de publicar encuestas que pueden responderse por cualquier ciudadano, sin necesidad de ningún tipo de identificación como en los casos anteriores. Estas encuestas son útiles para pulsar la opinión de la ciudadanía sobre temas en los que tiene que decidir el equipo de gobierno. No se trata de encuestas de base 
estadística, científicas, sino más bien de meros objetos de consulta que pueden servir para detectar posiciones más o menos generalizadas de la opinión pública, al estilo de las que desarrollan frecuentemente, por otro lado, los medios de comunicación.

Dentro de este portal de participación, también está a disposición de los ciudadanos, directamente, la consulta del registro municipal de asociaciones como medida para incentivar el asociacionismo entre la población y la realización de actividades en función de sus intereses. Como efecto colateral, esta publicación ha servido para que las propias asociaciones actualizasen los datos de registro que habían cambiado con el tiempo y no habían sido comunicados al Ayuntamiento en su momento.

\section{Conclusiones}

La puesta en marcha de la administración electrónica en el Ayuntamiento de Valladolid ha supuesto un esfuerzo importante, tanto desde el punto de visto económico, con ayuda de planes de inversión estatales, como tecnológico, por el cambio de plataformas de funcionamiento, y, sobre todo, organizativo, tratando de implicar a todo el colectivo de empleados públicos en un nuevo enfoque a la hora de prestar servicio al ciudadano.

Nos encontramos, por tanto, ante un verdadero proyecto de gestión del cambio, por más que queramos resaltar la parte tecnológica, donde el punto clave es el cambio de mentalidad a la hora de realizar el trabajo de todos los días, repensando, tanto las estructuras organizativas, como los procesos que se seguían antes de su entrada en funcionamiento.

Afortunadamente, este esfuerzo ha tenido su recompensa con la puesta en explotación satisfactoria del proyecto y también mediante distintos reconocimientos obtenidos por este proyecto de administración electrónica durante el año 2011.

Así, la sede electrónica se calificó como la mejor propuesta institucional en los premios Buscando el Norte Digital, organizados por un periódico regional. También, el informe España 2011, publicado por la Fundación Orange, sitúa al Ayuntamiento de Valladolid como el mejor situado en administración electrónica de todos los grandes municipios evaluados y en el estudio anual de 2011 sobre municipios que lleva a cabo al Organización de Consumidores y Usuarios, la administración electrónica del Ayunta- miento de Valladolid se consideró como la más avanzada de entre las 20 mayores ciudades del país.

\section{Referencias}

España, Jefatura del Estado (1992). Ley 30/1992, de 26 de Noviembre, de Régimen Jurídico de las Administraciones Públicas y del Procedimiento Administrativo Común. // Boletín Oficial del Estado. 285 (27 de noviembre de 1992) 40300-40319.

España, Jefatura del Estado (2002). Ley 34/2002, de 11 de julio, de Servicios de la Sociedad de la Información y de Comercio Electrónico. // Boletín Oficial del Estado. 285 (27 de noviembre de 1992) 40008-40319.

España, Jefatura del Estado (2003). Ley 59/2003, de 19 de diciembre, de Firma Electrónica. // Boletín Oficial del Estado. 166 (12 de julio de 2002) 25388 a 25403.

España, Jefatura del Estado (2007b). Ley 56/2007, de 28 de diciembre, de Medidas de Impulso de la Sociedad de la Información. // Boletín Oficial del Estado. 312 (29 de diciembre de 2007) 53701-53719.

España, Minsiterio de la Presidencia (2009). Real Decreto $1671 / 2009$, de 6 de noviembre, por el que se desarrolla parcialmente la Ley 11/2007, de 22 de junio, de Acceso Electrónico de los Ciudadanos a los Servicios Públicos. // Boletín Oficial del Estado. 278 (18 de noviembre de 2009) 97921-97948.

Ayuntamiento de Valladolid (2010). Reglamento de Administración Electrónica del Ayuntamiento de Valladolid, de 13 de diciembre de 2010. https://www.valladolid.gob.es/ es/normativa-sede/administracion-electronica-ayuntami ento-valladolid-reglamen.ficheros/18390-REG_ADMON_ ELECTRONICA.pdf

Portal de administración electrónica (PAE) del Gobierno de España: http://administracionelectronica.gob.es/ (consultado el 4-6-12).

UIT-T (2012). Norma X.509 de la UIT-T sobre certificados digitales para uso en infraestructuras de clave pública (PKI): http://es.wikipedia.org/wiki/Sitio_Webhttp://tools. ietf.org/rfc/rfc5280.txt (consultado el 4-6-12).

España, Jefatura del Estado (2007a). Ley 11/2007, de 22 de junio, de Acceso Electrónico de los Ciudadanos a los Servicios Públicos. // Boletín Oficial del Estado. 150 (23 de junio de 2007) 27150- 27166.

España, Ministerio de la Presidencia (2010). Real Decreto $3 / 2010$, de 8 de enero, por el que se regula el Esquema Nacional de Seguridad en el ámbito de la Administración Electrónica. // Boletín Oficial del Estado. 25 (29 de enero de 2010) 8089-8138

España, Ministerio de la Presidencia (2010). Real Decreto $4 / 2010$, de 8 de enero, por el que se regula el Esquema Nacional de Interoperabilidad en el ámbito de la Administración Electrónica. // Boletín Oficial del Estado. 25 (29 de enero de 2010) 8139-8156.

España, Jefatura del Estado (1999). Ley Orgánica 15/1999, de 13 de diciembre, de Protección de datos de Carácter Personal. // Boletín Oficial del Estado. 298 (13 de diciembre de 1999) 43088- 43099.

Enviado: 2012-04-12. Versión corregida: 2012-07-03. Aceptado: 2012-07-17. 
